\title{
The Real Problem(s) with Panpsychism
}

\author{
Anil K. Seth ${ }^{1,2}$ \\ ${ }^{1}$ Sackler Centre for Consciousness Science \\ School of Engineering and Informatics \\ University of Sussex \\ Brighton, BN1 9QJ, UK \\ ${ }^{2}$ Program for Brain, Mind, and Consciousness \\ Canadian Institute for Advanced Research (CIFAR), \\ Toronto, Ontario, M5G 1M1, Canada
}

\begin{abstract}
Panpsychism is the notion that consciousness is fundamental and ubiquitous. Defenders of panpsychism appeal, at least in part, to the apparent implausibility of materialist accounts of consciousness. However, materialist accounts are more resourceful than often assumed by proponents of panpsychism, while panpsychism has insurmountable problems of its own. The real problem with panpsychism is not that it seems crazy. It is that it explains nothing and does not generate testable predictions.
\end{abstract}

\subsection{Introduction}

Consciousness - subjective experience - has long puzzled philosophers and scientists. How can the 'what-it-is-likeness' of conscious experiences (qualia) be understood in relation to physical, material systems such as brains (and bodies)? The now standard statement of this question is in terms of David Chalmers' 'hard problem'. As he puts it [(Chalmers, 1995) p.201], "Why should physical processing give rise to a rich inner life at all? It seems objectively unreasonable that it should, and yet it does."

The materialist response to this challenge is that consciousness will, in the end, turn out to be a (perhaps emergent) property of particular arrangements of, or dynamical interactions among, physical elements, in ways that are yet to be discovered, and which may well require dramatically new concepts and methods. And the suspicion that this response will forever be inadequate plays a dominant role in motivating alternative metaphysical perspectives for considering consciousness, of which panpsychism is a prominent example. (The relevant history is more complicated, but this will do for now.) 
Panpsychism is the notion that consciousness is fundamental and ubiquitous in the natural world. This notion is sometimes caricatured, or misunderstood, as proposing that objects such as spoons, stones, and atoms have conscious experiences that are in some way relatable to the sorts of conscious experiences had by human beings. ${ }^{1}$ But sophisticated panpsychists do not say this at all. In his book Galileo's Error (Goff, 2019), Philip Goff notes that panpsychism does not propose that every 'thing' is conscious. Your socks may not be conscious, though - for Goff and other sophisticated panpsychists - they may be ultimately composed of things that are conscious. Equally, the kind of consciousness envisaged as being fundamental and ubiquitous is not consciousness 'like ours'. As Goff says, "If electrons have experience, then it is of some unimaginably simple form" (p.113; page numbers from here on refer to Goff, 2019). As we will see, the qualifier 'unimaginable' poses a challenge for the utility of panpsychism.

The immediate appeal of panpsychism appears to be that by building in consciousness from the 'ground up', there is no longer any hard problem to solve. But for Goff and others, there is a deeper motivation. Noting that scientific theories post-Galileo concern the disposition of entities - be they electrons or socks - to behave in particular ways, there is apparently nothing at all to be said about the intrinsic nature of anything. At the same time, conscious experiences seem to be all about intrinsic natures, and - on at least some views - resistant to characterisation in terms of dispositions. Goff traces the final step to the physicist Arthur Eddington: the proposal that consciousness is the intrinsic nature of matter, filling the unseemly hole at the centre of the scientific story (p.132). This is an elegant move, but being elegant does not mean being right, or being useful. ${ }^{2}$

\footnotetext{
${ }^{1}$ See https://neurobanter.com/2018/02/01/conscious-spoons-really-pushing-backagainst-panpsychism/ for a caricature of this caricature.

${ }^{2}$ A third, less frequently aired motivation is the 'argument from causation', which justifies panpsychism on the grounds that the phenomenology of will, motivation, and agency somehow reveals that all causation is mental, and therefore panpsychism must be true [see (Mørch, 2019) for a discussion]. This seems a particularly weak argument. For starters, the phenomenology of experiences of will, motivation, and agency no more justifies belief in the existence of actual 'mental causation' than the phenomenology of 'seeing red' justifies belief in external objectively existing 'redness'. See (Seth, 2021), chapter 11.
} 
Panpsychism - though it remains unpopular among many consciousness researchers ${ }^{3}$ and tends to attract overblown media coverage ${ }^{4}$ - is enjoying a day in the sun. Besides the influential philosophical expositions by Goff and others [see for example (Seager, 2020)], there are prominent neuroscientific theories of consciousness which indirectly imply some form of panpsychism. Here I am referring to Giulio Tononi's 'integrated information theory of consciousness' (IIT), according to which consciousness is identical to local maxima of irreducible integrated information (Tononi, Boly, Massimini, \& Koch, 2016; Tononi \& Koch, 2015). Because such maxima are found in many places, but not everywhere, the panpsychism implied by IIT is relatively restrained: consciousness is fundamental, but not ubiquitous. But it is panpsychism nonetheless. ${ }^{5}$

In this commentary, I will first examine the motivation for (at least some) panpsychist views, arguing that materialism is more resourceful than panpsychists tend to claim. I will then outline some weaknesses of panpsychism, focusing on explanatory power and empirical testability. I will finish by recognising that both consciousness and matter are more complicated than they might at first appear.

\subsection{The resourcefulness of materialism}

What should a successful materialist account of consciousness accomplish? At first glance, one might be tempted to set the bar at a full solution to the hard problem of consciousness. On this view, the path towards a successful account might consist of increasingly detailed attempts to directly address this problem.

An alternative view is that a materialist account of consciousness is best pursued, not by addressing the hard problem 'head on', but instead by

\footnotetext{
${ }^{3}$ In a recent survey of 232 attendees of annual meetings of the Association for the Scientific Study of Consciousness - a meeting which attracts neuroscientists, psychologists, and philosophers - a large majority agreed with the proposition that a complete biophysical explanation of consciousness is either definitely or probably possible, and an overwhelming majority thought that rocks and thermostats lack consciousness; see https://psyarxiv.com/8mbsk/.

${ }^{4}$ For example: https://www.dailymail.co.uk/news/article-9820481/Scientistsdebating-bizarre-theory-consciousness-including-inanimate-objects.html

${ }_{5}$ The non-ubiquity implied by IIT is quite specific. Feedforward neural networks, for example, are not conscious, regardless of how complex their input-output mappings might be. And when a macroscopic entity such as a human brain is conscious, in virtue of instantiating irreducible maxima of integrated information, the parts that make it up - neurons, for example - lack consciousness. See (Tononi \& Koch, 2015).
} 
building increasingly sturdy explanatory bridges between properties of physical (e.g., neural) mechanisms and properties of conscious experience. The latter are both functional (i.e., those which are readily explained in terms of mechanisms that produce effects) and, critically, phenomenological. (Note that I am not claiming that these properties are independent - it is likely they are not independent.) Accounting for the phenomenological properties of consciousness means, at least in part, explaining why a particular experience (or class of experiences) is the way it is, and not some other way: why the experience of redness is not like the experience of blueness, or jealousy, or toothache.

I have called this (with tongue a little bit in cheek) the 'real problem' of consciousness: how to explain, predict and control the various properties of consciousness in terms of physical processes in the brain and body (Hohwy \& Seth, 2020; Seth, 2016, 2021). The real problem is distinct from the hard problem because it is not, at least not in the first instance, about explaining why and how consciousness is part of our universe. (Already you can see that panpsychism does not address itself to the real problem.) And it is distinct from Chalmers' panoply of so-called 'easy problems' because it does not sweep the experiential, subjective aspects of consciousness away under the carpet.

The real problem approach is by no means new - precursors and complementary perspectives include neurophenomenology (Varela, Thompson, \& Rosch, 1993), and Chalmers' own 'mapping problem' (Chalmers, 1996). Another useful way to think of this approach is as generalizing the popular and empirically productive notion of 'neural correlates' of consciousness to that of 'explanatory' or 'systematic' correlates of consciousness (Hohwy \& Seth, 2020; Seth, 2009).

Worries about the adequacy of materialism do not apply to the real problem, as they might potentially apply to the hard problem. ${ }^{6}$ There are many ways to leverage the resources of materialism to develop and test explanatory bridges between mechanism and phenomenology. Two examples from my own work include the use of predictive models to account for the characteristic phenomenology of different perceptual experiences (Hohwy \& Seth, 2020; Seth, 2014), and the development of quantitative measures of emergence to account for the ways in which unified conscious experiences seem to be more than the sum of their parts

${ }^{6}$ Such worries are often misplaced even when applied to the hard problem. The infamous zombie argument against materialism, for example, is intrinsically weak because it is a conceivability argument. 
(Barnett \& Seth, 2021; Rosas et al., 2020). Rather than reviewing the details of these and other examples (Seth, 2021), I will instead highlight three more general points.

First, explanation, prediction, and control are the criteria by which many scientific enterprises are assessed. To be successful, it is not always essential - at least not at first - for a science of X to account for the presence of $X$ in the universe in the first place. Physics has not (yet) accounted for the presence of the universe, the properties of which it does such a brilliant job of explaining.

Second, the explanations provided by a science of X need not be intuitively satisfying. Quantum mechanics, for example, is not intuitively satisfying, which is putting it mildly since there is no consensus on what it even means (we'll come back to this). The case in which X equals 'consciousness' should not imply a different standard for intuitive satisfaction. Indeed, the very fact that we instantiate phenomenological properties ourselves might mean that a scientifically satisfactory account of consciousness will never generate the intuitive feeling that 'ah yes, this is right, it has to be this way'. These first two points mean that the supposed inadequacy of materialism that motivates panpsychism in part results from setting the explanatory bar too high.

Third, an intuitively satisfying and hard-problem-complete account of consciousness is not ruled out by the real problem approach. It is just not guaranteed by it. It could well be, and I suspect that it will be, that addressing the real problem will dissolve rather than outright solve the hard problem. I have motivated this prospect [like others, including especially Patricia Churchland (Churchland, 1996)], by appealing to the analogy with the scientific understanding of life.

Briefly, the vitalist notion that life could not be explained in terms of biophysical mechanisms was neither directly solved (by finding the elusive 'spark of life') nor eradicated (by discovering that life does not exist). It was dissolved when biologists stopped treating life as one big scary mystery, and instead started accounting for (i.e., explaining, predicting, and controlling) the properties of living systems (reproduction, homeostasis, and so on) in terms of physical and chemical processes. We still don't understand everything about life, but what seemed at one time beyond the reach of materialism no longer does. By analogy, the fact that consciousness seems hard-problem mysterious now, with the tools and concepts we have now, does not mean it will always seem hard-problem 
mysterious - and the best way forward is to build the sturdiest explanatory bridges that we can, and see how far we get.

This brings us back to Goff, who notes my use of this analogy in his book, and is unmoved. Goff argues that the problem with the analogy is that sometimes science progresses otherwise. He notes that Darwin's theory of evolution by natural selection solved an apparent mystery - the nature and origin of biological species - through a dramatic insight, rather than through incremental dissolution (pp.7-8). This is a curious argument. I am not claiming that dramatic insights do not matter or never happen. To the contrary, it is likely that dramatic insights into how explanatory bridges can be built will be needed if the real problem approach is to succeed. Moreover, Darwin's insight did not come out of nowhere. Besides being independently discovered by Alfred Russell Wallace, a strong case can be made that the conditions needed for Darwin (and Wallace) to have their insights depended on a long history of gradual alterations in how people thought about incremental change and the depth of historical time - from Charles Lyell's geology, to the breakdown of previously fixed social classes (Levins \& Lewontin, 1987).

A more common objection is that life and consciousness are simply too different for the analogy to hold water. The usual argument here is that living systems - however mysterious they may once have seemed - are objectively describable, whereas consciousness is by definition a subjective phenomenon. Because consciousness is intrinsically subjective, the argument goes, mechanistic explanations are inadequate in a more problematic sense than was the case for life.

We can concede that this difference is important, without conceding the utility of the analogy, for two reasons. First, the analogy is useful primarily in an historical sense, through underlining the imprudence of pronouncing the insufficiency of mechanistic, materialist accounts of a putatively mysterious phenomenon before such accounts have been taken as far as they can go. Second, the difference should not be overstated. The subjective mode of existence of conscious experiences mostly means the relevant data are difficult to collect and compare. This is challenging (Phillips, 2018), but it does not exclude the possibility of a successful science of consciousness. Methodological developments in (for example) applied or computational phenomenology hold great promise in this regard (Suzuki, Roseboom, Schwartzman, \& Seth, 2017), as does the practice of phenomenology more generally. As John Searle noted long ago, it is possible to be 
epistemologically objective about something that is ontologically subjective (Searle, 1998); see also (Dennett, 1991).

Goff raises one other objection to materialism which is worth covering off here. He argues that "physical science tries to give a purely quantitative characterisation of reality, a description involving only mathematical terms" (p.68). Noting that conscious experiences involve qualitative concepts such as "yellow" or "sour", Goff concludes that "[m]aterialists who claim both that reality can be exhaustively described in the quantitative language of physical science and that there is quality-rich consciousness contradict themselves" (p.68).

There is no contradiction. A successful science of consciousness does not require exhaustive description (it is not entirely clear what 'exhaustive' means here - one suspects it might mean something close to instantiation, or perhaps to 'a priori scrutability'7) - it requires sufficient description for the purposes of explanation, prediction, and control. In addition, quantitative elements can be readily introduced into phenomenological methods - as can already be seen in examples such as quantitative colour spaces (Bird, Berens, Horner, \& Franklin, 2014). Finally, many successful scientific explanations operate with qualitative rather than, or as well as, quantitative concepts. Darwin's theory - highlighted by Goff as a paragon of materialist science - provides one striking example of such an explanation. (Note that the meaning of 'qualitative' is different in evolution than in consciousness; this is a distinction that Goff glosses over.)

Altogether, there is much more to materialism than the proposal of 'brute identities' between the physical and the phenomenal that Goff finds "very unsatisfying" (p.108). The real problem approach promises to repair Galileo's error - an error elegantly diagnosed by Goff as that of removing sensory qualities from the remit of science - without leaning on brute identities, and, equally, without succumbing to the temptations of easy big 'solutions' like panpsychism. Put simply, the best strategy is to identify and account for properties of consciousness, and repeat. Following this strategy may ultimately deliver identities between the physical and the phenomenal - but, given their ability to explain, predict, and control - these identities would no longer be 'brute'.

\footnotetext{
${ }^{7} A$ priori scrutability, as articulated by (Chalmers, 2012), is a way of making precise the notion that a phenomenon is in principle fully deducible from a description. Understood this way, an exhaustive description would be an endpoint of either fully solving, or fully dissolving, the hard problem.
} 


\subsection{The impotence of panpsychism}

What if we took panpsychism seriously, and diverted all our intellectual resources to pursuing a panpsychist solution to the problem of consciousness?

According to Goff, this would mainly involve devising solutions to the socalled 'combination problem'. Broadly, this is the problem of how to get from little conscious things (such as electrons) to big conscious things (such as philosophers). Goff sketches a variety of philosophical approaches to closing this 'panpsychist gap', which may or may not make progress (p.147). But the combination problem is only a problem if you assume panpsychism in the first place. While other (perhaps materialist) approaches may face related problems - such as explaining the unity of consciousness (Bayne, 2010) - these are substantially different from the combination problem. The panpsychist gap is therefore different from the widely recognised 'explanatory gap' in consciousness research (Levine, 1983), which can be understood as a relevant challenge from a variety of theoretical and metaphysical starting points. In other words, the main problem that the panpsychist methodology addresses is a problem of its own making.

This observation leads to the related point that panpsychism offers no useful insight into consensus explanatory targets for consciousness science. Asserting that consciousness is fundamental and ubiquitous does nothing to shed light on why an experience of blueness is the way it is, and not some other way. Nor does it explain anything about the possible functions of consciousness, nor why consciousness is lost in states such as dreamless sleep, general anaesthesia, and coma. ${ }^{8}$ In acknowledging that the putative consciousness of a single electron will be unimaginably different from the consciousness of a human being (p.113), we find the implicit concession that adopting panpsychism has no implications for explaining anything about subjective properties of consciousness as they pertain to conscious organisms.

\footnotetext{
${ }^{8}$ Goff wonders whether such states might induce a disruption of subsequent memory, rather than an interruption of consciousness itself (p.141). However, while some reported absences of consciousness may reflect amnesia, to defend panpsychism on the basis of there being no explanatorily interesting difference in consciousness between coma and wakeful awareness is a high price to pay.
} 
Solving the combination problem does not address this criticism, since the combination problem concerns how already-conscious things are combined, or dissociated, into other conscious things. At most, it would offer a highly indirect way of explaining the basis of characteristic properties of consciousness, real problem style, but to which the core panpsychist assumption adds nothing besides other, uniquely panpsychist, problems.

Worst of all for panpsychism is that it is not itself testable, and that it does not lead to testable predictions. The challenges are overwhelming, going far beyond the difficulties of relating subjective reports to objective (e.g., neural) data. How can one begin to assess whether an electron is conscious? How does assuming panpsychism lead to any new testable predictions about consciousness whatsoever, whether in electrons, octopuses, or philosophers? The absence of responses to questions like these in Goff's otherwise comprehensive manifesto is striking.

One possible response to the testability challenge is that theories which indirectly imply some form of panpsychism may generate testable predictions. Here, again, I am thinking of IIT. However, in the case of IIT, the panpsychist interpretation makes experimental predictions less testable, rather than more testable. Specifically, empirical measurements of the core quantity of $\Phi$ (Phi) are generally infeasible on the 'strong' (panpsychist) version of IIT, according to which one needs to measure all the states a system could potentially be in (its maximum entropy distribution). However, there are 'weaker' readings of IIT, better aligned with the real problem approach, in which integrated information is treated as an explanatory correlate linking properties of neural mechanisms to pervasive phenomenological features of global conscious states. Measures of $\Phi$ based on the weaker reading only require knowledge of the actual occurent dynamics of the system (its empirical distribution), and so are empirically tractable (Barrett \& Seth, 2011; Mediano, Seth, \& Barrett, 2019). ${ }^{9}$

Ultimately, it is precisely the broader appeal of panpsychism, which Goff traces to Arthur Eddington, that guarantees its empirical impotence. Associating consciousness with the intrinsic - rather than the dispositional

\footnotetext{
${ }^{9}$ One intriguing prediction flowing from IIT is that neurons may contribute to conscious experiences if they are inactive, but not if they are explicitly inactivated (Tononi et al., 2016). This prediction is difficult, though perhaps not impossible, to test. However, should the experiments turn out to be feasible and to favour IIT, this by no means rules out non-panpsychist accounts of the data.
} 
- nature of things guarantees that the property of 'being conscious' cannot itself make an experimentally measurable difference to anything, since to do so would require a dispositional aspect. [The flip side of this conclusion is also worrying. As Sean Carroll points out in his commentary (Carroll, 2021), if the property of being conscious were to make a difference to things at a fundamental level, this would mean modifying the known laws of physics, which is not to be undertaken lightly.]

There is something ironic in Arthur Eddington's central role in all this, since Eddington's acclaim was largely due to his formulating testable predictions deriving from Einstein's general theory of relativity, and it was the dramatic experimental validation of these predictions that led to Einstein's theory playing a dominant role ever since (Kennefick, 2019). The contrast with panpsychism is clear: the success of modern physics is entirely due to its weird ideas - be they from relativity or quantum mechanics - being experimentally testable.

\subsection{What is matter, anyway}

Returning to materialism, I've argued against underestimating its powers from two related perspectives: shifting the explanatory target from the hard problem to the real problem, and noting the rich resources available for building explanatory bridges from neural mechanisms to properties of phenomenology. But materialism is fundamentally a claim about matter in general, rather than neurons in particular, and it is worth bearing in mind that the fundamental nature of matter is far from being understood.

Quantum mechanics - our current best theory of matter, and one that has stood up to every experimental interrogation that has been thrown at it admits several interpretations, each bizarre, all controversial, and every one of which undermines the naïve materialistic picture of a universe ultimately consisting of particles of various kinds interacting via forces. It could be that a richer picture of matter itself might further deepen the resources of materialism. For example, the 'relational' interpretation of quantum mechanics argues that the fundamental nature of matter is in the form of interactions (Rovelli, 1996). It is conceivable, though by no means guaranteed, that adopting such a perspective may in the long run change one's views about the possibilities of materialist explanations - not only of consciousness, but of many other phenomena too. [See Carlo Rovelli's enchanting Helgoland for something along these lines (Rovelli, 2021); see also Rovelli, this volume]. 
I stress that I am not falling into the lazy and false syllogism that consciousness and quantum mechanics are both mysterious, so therefore they must be related. Nor am I condoning theories that propose a specific basis for consciousness in terms of quantum mechanical processes (Hameroff \& Penrose, 2014), nor those that propose that consciousness brings the classical world into existence by collapsing the wave function (Chalmers \& McQueen, (in press); Wigner, 1961). There is plenty of work to be done in building explanatory bridges between mechanism and phenomenology using the uncontroversial tools of classical physics, neuroscience, and psychology, and this project may well be sufficient to dissolve the hard problem. My argument is simply that respecting the complexities of both consciousness and matter provides yet more reason to believe that resorting to panpsychism reflects a failure of imagination rather than an insight into necessity.

\subsection{Summary}

Goff offers a four-point manifesto for a post-Galilean science of consciousness (p.174). I'm completely on board with the first three: realism about consciousness, empiricism, and anti-dualism. The fourth - a panpsychist methodology - is where I get off the bus. Panpsychism explains nothing, cannot be tested in itself, does not lead to testable predictions, and may actively discourage the generation of such predictions. It is a seductive easy-out to the hard problem, and there is no need for it.

A real problem approach, rooted in materialism, but going far beyond establishing mere correlations or prematurely proposing brute identities, has more than enough gumption to explain, predict, and control properties of consciousness in terms of their underlying mechanisms. It remains to be seen whether following this approach will fully dissolve the hard problem, or whether residues of mystery will persist, but the journey on this particular bus is going to be beautiful and enlightening, and there are plenty of tickets still for sale.

Acknowledgements. I am grateful to Philip Goff both for writing his accessible and illuminating book, for inviting me to write this commentary. Many thanks to Tim Bayne for comments on a draft of this paper. I am also grateful to the Dr. Mortimer and Theresa Sackler Foundation and to the Canadian Institute for Advanced Research (CIFAR) Program on Brain, Mind, and Consciousness. 


\section{References:}

Barnett, L. C., \& Seth, A. K. (2021). Dynamical independence: discovering emergent macroscopic processes in complex dynamical systems.

Barrett, A. B., \& Seth, A. K. (2011). Practical measures of integrated information for timeseries data. PLoS Comput Biol, 7(1), e1001052. doi:10.1371/journal.pcbi.1001052

Bayne, T. (2010). The unity of consciousness. Oxford: Oxford University Press.

Bird, C. M., Berens, S. C., Horner, A. J., \& Franklin, A. (2014). Categorical encoding of color in the brain. Proc Natl Acad Sci U S A, 111(12), 4590-4595.

doi:10.1073/pnas.1315275111

Carroll, S. (2021). Consciousness and the laws of physics. Journal of Consciousness Studies.

Chalmers, D. J. (1995). Facing up to the problem of consciousness. Journal of Consciousness Studies, 23(3), 200-219.

Chalmers, D. J. (1996). The conscious mind: In search of a fundamental theory. New York, NY: Oxford University Press.

Chalmers, D. J. (2012). Constructing the world. Oxford: Oxford University Press.

Chalmers, D. J., \& McQueen, K. ((in press)). Consciousness and the collapse of the wave function. In S. Gao (Ed.), Consciousness and Quantum Mechanics. Oxford: Oxford University Press.

Churchland, P. S. (1996). The hornswoggle problem. Journal of Consciousness Studies, 3(5-6), 402-408.

Dennett, D. C. (1991). Consciousness Explained. Boston, MA: Little, Brown, and London.

Goff, P. (2019). Galileo's Error: Foundations for a New Science of Consciousness. London: Rdier.

Hameroff, S., \& Penrose, R. (2014). Consciousness in the universe: a review of the 'Orch OR' theory. Phys Life Rev, 11(1), 39-78. doi:10.1016/j.plrev.2013.08.002

Hohwy, J., \& Seth, A. K. (2020). Predictive processing as a systematic basis for identifying the neural correlates of consciousness. Philosophy and the Mind Sciences, 1(2), 3.

Kennefick, D. (2019). No shadow of a doubt: The 1919 eclipse that confirmed Einstein's theory of relativity. Princeton, NJ: Princeton University Press.

Levine, J. (1983). Materialism and qualia: The explanatory gap. Pacific Philosophical Quarterly, 64, 354-361.

Levins, R., \& Lewontin, R. C. (1987). The dialectical biologist. Cambridge, MA: Harvard University Press.

Mediano, P. A. M., Seth, A. K., \& Barrett, A. B. (2019). Measuring integrated information: comparison of candidate measures in theory and simulation. Entropy, 21(1), 17. doi:https://doi.org/10.3390/e21010017

Mørch, H. H. (2019). The argument for panpsychism from experience of causation. In W. Seager (Ed.), The Routlledge Hanbook of Panpsyschism: Routledge.

Phillips, I. (2018). The methodological puzzle of phenomenal consciousness. Philos Trans $R$ Soc Lond B Biol Sci, 373(1755). doi:10.1098/rstb.2017.0347

Rosas, F., Mediano, P. A. M., Jensen, H. J., Seth, A. K., Carhart-Harris, R., \& Bor, D. (2020). Reconciling emergences: An information-theoretic approach to identify causal emergence in multivariate data. PLoS Comput Biol.

Rovelli, C. (1996). Relational quantum mechanics. International journal of theoretical physics, 35, 1637-1678.

Rovelli, C. (2021). Helgoland. London: Allen Lane. 
Seager, W. (Ed.) (2020). The Routledge Handbook of Panpsychism: Routledge.

Searle, J. (1998). Mind, Language, and Society: Doing Philosophy in the Real World. New York, NY: Basic Books.

Seth, A. K. (2009). Explanatory correlates of consciousness: Theoretical and computational challenges. Cognitive Computation, 1(1), 50-63.

Seth, A. K. (2014). A predictive processing theory of sensorimotor contingencies: Explaining the puzzle of perceptual presence and its absence in synesthesia. Cogn Neurosci, 5(2), 97-118. doi:10.1080/17588928.2013.877880

Seth, A. K. (2016). The real problem. Aeon. Retrieved from https://aeon.co/essays/the-hardproblem-of-consciousness-is-a-distraction-from-the-real-one website:

Seth, A. K. (2021). Being You: A New Science of Consciousness. London: Faber \& Faber.

Suzuki, K., Roseboom, W., Schwartzman, D. J., \& Seth, A. K. (2017). A Deep-Dream Virtual Reality Platform for Studying Altered Perceptual Phenomenology. Sci Rep, 7(1), 15982. doi:10.1038/s41598-017-16316-2

Tononi, G., Boly, M., Massimini, M., \& Koch, C. (2016). Integrated information theory: from consciousness to its physical substrate. Nat Rev Neurosci, 17(7), 450-461. doi:10.1038/nrn.2016.44

Tononi, G., \& Koch, C. (2015). Consciousness: here, there and everywhere? Philos Trans $R$ Soc Lond B Biol Sci, 370(1668). doi:10.1098/rstb.2014.0167

Varela, F. J., Thompson, E., \& Rosch, E. (1993). The embodied mind: Cognitive science and human experience. Cambridge, MA: MIT Press.

Wigner, E. (1961). Remarks on the mind-body problem. In I. J. Good (Ed.), The Scientist Speculates. 